\title{
A Central Limit Theorem for Random Walks on the Dual of a Compact Grassmannian
}

\author{
Margit RÖSLER ${ }^{\dagger}$ and Michael VOIT ${ }^{\ddagger}$ \\ $\dagger$ Institut für Mathematik, Universität Paderborn, \\ Warburger Str. 100, D-33098 Paderborn, Germany \\ E-mail: roesler@math.upb.de \\ $\ddagger$ Fakultät für Mathematik, Technische Universität Dortmund, \\ Vogelpothsweg 87, D-44221 Dortmund, Germany \\ E-mail: michael.voit@math.uni-dortmund.de
}

Received October 14, 2014, in final form February 03, 2015; Published online February 10, 2015 http://dx.doi.org/10.3842/SIGMA.2015.013

\begin{abstract}
We consider compact Grassmann manifolds $G / K$ over the real, complex or quaternionic numbers whose spherical functions are Heckman-Opdam polynomials of type $B C$. From an explicit integral representation of these polynomials we deduce a sharp Mehler-Heine formula, that is an approximation of the Heckman-Opdam polynomials in terms of Bessel functions, with a precise estimate on the error term. This result is used to derive a central limit theorem for random walks on the semi-lattice parametrizing the dual of $G / K$, which are constructed by successive decompositions of tensor powers of spherical representations of $G$. The limit is the distribution of a Laguerre ensemble in random matrix theory. Most results of this paper are established for a larger continuous set of multiplicity parameters beyond the group cases.
\end{abstract}

Key words: Mehler-Heine formula; Heckman-Opdam polynomials; Grassmann manifolds; spherical functions; central limit theorem; asymptotic representation theory

2010 Mathematics Subject Classification: 33C52; 43A90; 60F05; 60B15; 43A62; 33C80; $33 \mathrm{C} 67$

\section{Introduction}

For Riemannian symmetric spaces $G / K$ of the compact or non-compact type, there is a wellknown contraction principle which states that under suitable scaling, the spherical functions $\varphi_{\lambda}$ of $G / K$ tend to the spherical functions $\psi_{\lambda}$ of the tangent space of $G / K$ in the base point, which is a symmetric space of the flat type:

$$
\lim _{n \rightarrow \infty} \varphi_{n \lambda}(\exp (x / n))=\psi_{\lambda}(x) .
$$

See [4] and, for a more recent account, [2]. This curvature limit, also known as Mehler-Heine formula, extends to the more general setting of hypergeometric functions associated with root systems, which converge under rescaling to generalized Bessel functions. This is proven in [6] by a limit transition in the Cherednik operators; see also [2] for a different approach. In the compact rank one case, the contraction principle is a weak version of the classical Hilb formula for Jacobi polynomials (see [27, Theorem 8.21.12]), which provides in addition a precise estimate on the rate of convergence. In this paper, we prove a Mehler-Heine formula with a precise estimate on the error term for a certain class of orthogonal polynomials associated with root systems, which in particular encompasses the spherical functions of compact Grassmannians. This result is a "compact" analogue of Theorem 5.4 in [26], which gives a scaling limit with error bounds for hypergeometric functions in the dual, non-compact setting. In the second part of the paper, we 
shall use the Mehler-Heine formula 2.4 in order to establish a central limit theorem for random walks on the semi-lattice of dominant weights parametrizing the unitary dual of a compact Grassmannian.

To become more precise, we consider the compact Grassmannians $\mathcal{G}_{p, q}(\mathbb{F})=G / K$ over one of the (skew-) fields $\mathbb{F}=\mathbb{R}, \mathbb{C}, \mathbb{H}$, with $G=S U(p+q, \mathbb{F})$ and $K=S(U(q, \mathbb{F}) \times U(p, \mathbb{F}))$, where $p \geq q \geq 1$. Via polar decomposition of $G$, the double coset space $G / / K=\{K g K: g \in G\}$ may be topologically identified with the fundamental alcove

$$
A_{0}:=\left\{x=\left(x_{1}, \ldots, x_{q}\right) \in \mathbb{R}^{q}: \frac{\pi}{2} \geq x_{1} \geq x_{2} \geq \cdots \geq x_{q} \geq 0\right\},
$$

with $x \in A_{0}$ being identified with the matrix

$$
a_{x}=\left(\begin{array}{ccc}
\cos \underline{x} & -\sin \underline{x} & 0 \\
\sin \underline{x} & \cos \underline{x} & 0 \\
0 & 0 & I_{p-q}
\end{array}\right) .
$$

Here we use the diagonal matrix notation $\underline{x}=\operatorname{diag}\left(x_{1}, \ldots, x_{q}\right)$, and the functions sin, $\cos$ are understood component-wise. For details, see [23, Theorem 4.1]. The spherical functions of $\mathcal{G}_{p, q}(\mathbb{F})$ can be viewed as Heckman-Opdam polynomials of type $B C_{q}$, which are also known as multivariable Jacobi polynomials. They may be described as follows: denote by $F_{B C}(\lambda, k ; \cdot)$ the Heckman-Opdam hypergeometric function associated with the root system

$$
R=2 B C_{q}=\left\{ \pm 2 e_{i}, \pm 4 e_{i}: 1 \leq i \leq q\right\} \cup\left\{ \pm 2 e_{i} \pm 2 e_{j}: 1 \leq i<j \leq q\right\} \subset \mathbb{R}^{q},
$$

with spectral variable $\lambda \in \mathbb{C}^{q}$ and multiplicity parameter $k=\left(k_{1}, k_{2}, k_{3}\right) \in \mathbb{R}^{3}$ corresponding to the roots $\pm 2 e_{i}, \pm 4 e_{i}$ and $2\left( \pm e_{i} \pm e_{j}\right)$. Fix the positive subsystem

$$
R_{+}=\left\{2 e_{i}, 4 e_{i}, 1 \leq i \leq q\right\} \cup\left\{2 e_{i} \pm 2 e_{j}, 1 \leq i<j \leq q\right\}
$$

and the associated semi-lattice of dominant weights,

$$
P_{+}=\left\{\lambda \in(2 \mathbb{Z})^{q}: \lambda_{1} \geq \lambda_{2} \geq \cdots \geq \lambda_{q} \geq 0\right\} .
$$

Then the set of spherical functions of $\mathcal{G}_{p, q}(\mathbb{F})$ is parametrized by $P_{+}$and consists of the functions

$$
\varphi_{\lambda}^{p}\left(a_{x}\right)=F_{B C}\left(\lambda+\rho_{p}, k(p), i x\right)=: R_{\lambda}^{p}(x), \quad \lambda \in P_{+}
$$

with multiplicity parameter

$$
k(p)=(d(p-q) / 2,(d-1) / 2, d / 2),
$$

where $d=\operatorname{dim}_{\mathbb{R}} \mathbb{F} \in\{1,2,4\}$ and

$$
\rho_{p}=\frac{1}{2} \sum_{\alpha \in R_{+}} k_{\alpha} \alpha=\sum_{i=1}^{q}\left(\frac{d}{2}(p+q+2-2 i)-1\right) e_{i} .
$$

The functions $R_{\lambda}^{p}$ are the Heckman-Opdam polynomials associated with the root system $R$ (called Jacobi polynomials in the following) and with multiplicity $k(p)$, normalized according to $R_{\lambda}^{p}(0)=1$. We refer to $[12,13,22]$ for Heckman-Opdam theory in general, and to [23] and the references cited there for the connection with spherical functions in the compact $B C$ case. Notice that our notion of $F_{B C}$ coincides with that of Heckman, Opdam and [25, 26], while it differs from the geometric notion in [23]. Theorem 4.3 of [23] corresponds to (1.1).

In Theorem 4.2 of [23], the product formula for spherical functions of $(G, K)$ was written as a formula on $A_{0}$ and analytically extended to a product formula for the Jacobi polynomials $R_{\lambda}^{p}$ 
with multiplicity $k(p)$ corresponding to arbitrary real parameters $p>2 q-1$. This led to three continuous series of positive product formulas for Jacobi polynomials corresponding to $\mathbb{F}=\mathbb{R}, \mathbb{C}, \mathbb{H}$ and to associated commutative hypergroup structures on $A_{0}$; see [15] and [3] for the notion of hypergroups. Using a Harish-Chandra-type integral representation for the $R_{\lambda}^{p}$, we shall derive a Mehler-Heine formula with a precise asymptotic estimate for the Jacobi polynomials $R_{\lambda}^{p}$ in terms of Bessel functions associated with root system $B_{q}$ on the Weyl chamber

$$
C=\left\{x=\left(x_{1}, \ldots, x_{q}\right) \in \mathbb{R}^{q}: x_{1} \geq \cdots \geq x_{q} \geq 0\right\} .
$$

This Mehler-Heine formula will be the key ingredient for the main result of the present paper, a central limit theorem for random walks on the semi-lattice $P_{+}$, which parametrizes the spherical unitary dual of $G / K$. To explain this CLT, let us first recall that via the GNS representation, the spherical functions $\varphi_{\lambda}, \lambda \in P_{+}$of $(G, K)$, which are necessarily positive definite, are in a oneto-one correspondence with the (equivalence classes of) spherical representations $\left(\pi_{\lambda}, H_{\lambda}\right)$ of $G$, that is those irreducible unitary representations of $G$ whose restriction to $K$ contains the trivial representation with multiplicity one, see [9] or [14, Chapter IV]. The decomposition of tensor products of spherical representations into their irreducible components leads to a probability preserving convolution $*_{d, p}$ and finally a Hermitian hypergroup structure on the discrete set $P_{+}$; see [7] and [18]. Following, e.g., [3, 29, 32], we introduce random walks $\left(S_{n}^{d, p}\right)_{n \geq 0}$ on $P_{+}$associated with $*_{d, p}$ and derive some limit theorems for $n \rightarrow \infty$. The main result of this paper will be the Central Limit Theorem 3.12. This CLT implies the following result for $\mathcal{G}_{p, q}(\mathbb{F})=G / K$ :

Theorem 1.1. Let $\left(\pi_{\lambda}, H_{\lambda}\right)$ be a non-trivial spherical representation of $G$ associated with $\lambda \in$ $P_{+} \backslash\{0\}$. Let $u_{\lambda} \in H_{\lambda}$ be $K$-invariant with $\left\|u_{\lambda}\right\|=1$. For each $n \in \mathbb{N}$, decompose the $n$-fold tensor power $\left(\pi_{\lambda}^{\otimes, n}, H_{\lambda}^{\otimes, n}\right)$ into its finitely many irreducible unitary components

$$
\left(\pi_{\lambda}^{\otimes, n}, H_{\lambda}^{\otimes, n}\right)=\left(\bigoplus_{\tau_{n}} \pi_{\tau_{n}}, \bigoplus_{\tau_{n}} H_{\tau_{n}}\right)
$$

where the components are counted with multiplicities. Consider the orthogonal projections $p_{\tau_{n}}$ : $H_{\lambda}^{\otimes, n} \rightarrow H_{\tau_{n}}$ and a $P_{+}$-valued random variable $X_{n, \lambda}$ with the finitely supported distribution

$$
\sum_{\tau_{n}}\left\|p_{\tau_{n}}\left(u_{\lambda}^{\otimes, n}\right)\right\|^{2} \delta_{\tau_{n}} \in M^{1}\left(P_{+}\right)
$$

with the point measures $\delta_{\tau_{n}}$ at $\tau_{n}$. Then, for $n \rightarrow \infty$,

$$
\frac{X_{n, \lambda}}{m(\lambda) \sqrt{n}}
$$

tends in distribution to

$$
d \rho_{d, p}(x)=c_{d, p}^{-1} \prod_{j=1}^{q} x_{j}^{d(p-q+1)-1} \prod_{1 \leq i<j \leq q}\left(x_{i}^{2}-x_{j}^{2}\right)^{d} e^{-\left(x_{1}^{2}+\cdots+x_{q}^{2}\right) / 2} d x \in M^{1}(C)
$$

with a suitable normalization $c_{d, p}$. Notice that the probability measure $d \rho_{d, p}$ is the distribution of a Laguerre ensemble on $C$. The modified variance parameter $m(\lambda)>0$ is a second order polynomial in $\lambda$ and given explicitly in Lemma 3.3 below.

For $q=1$, the Central Limit Theorem 3.12 has a long history as a CLT for random walks on $\mathbb{Z}_{+}$whose transition probabilities are related to product linearizations of Jacobi polynomials. This includes random walks on the duals of $\mathrm{SU}(2)$ and $(\mathrm{SO}(n), \mathrm{SO}(n-1))$ in [8] and [11]. See also [29] for further one-dimensional cases. For $q \geq 2$ our results are very closely related to the work [5] of Clerc and Roynette on duals of compact symmetric spaces. For a survey on limits for spherical functions and CLTs in the non-compact case for $q=1$ we refer to [31]. 


\section{A Mehler-Heine formula}

In this section we derive a Mehler-Heine formula for the Jacobi polynomials $R_{\lambda}^{p}\left(\lambda \in P_{+}\right)$, describing the approximation of these polynomials in terms of Bessel functions with a precise error bound. Our result will be based on Laplace-type integrals for the Jacobi polynomials and the associated Bessel functions, where we treat the group cases with integers $p \geq q$ as well as the case $p \in \mathbb{R}$ with $p \geq 2 q-1$ beyond the group case. The integral representation for $R_{\lambda}^{p}$ below is a special case of a more general Harish-Chandra integral representation for hypergeometric functions $F_{B C}$ in [26]. To start with, let us introduce some notation:

Let $H_{q}(\mathbb{F})=\left\{x \in M_{q}(\mathbb{F}): x^{*}:=\bar{x}^{t}=x\right\}$ denote the space of Hermitian matrices over $\mathbb{F}$, and denote by $\Delta(x)$ the determinant of $x \in H_{q}(\mathbb{F})$, which may be defined as the product of (right) eigenvalues of $x$. We mention that for $\mathbb{F}=\mathbb{H}$, this is just the Moore determinant, which coincides with the Dieudonné determinant if $x$ is positive semi-definite, see, e.g., [1]. On $H_{q}(\mathbb{F})$, we consider the power functions

$$
\Delta_{\lambda}(a):=\Delta_{1}(a)^{\lambda_{1}-\lambda_{2}} \cdots \Delta_{q-1}(a)^{\lambda_{q-1}-\lambda_{q}} \Delta_{q}(a)^{\lambda_{q}}, \quad \lambda \in \mathbb{C}^{q}
$$

with the principal minors $\Delta_{r}(a)=\operatorname{det}\left(\left(a_{i j}\right)_{1 \leq i, j \leq r}\right)$ of the matrix $a=\left(a_{i j}\right)_{1 \leq i, j \leq q} \in H_{q}(\mathbb{F})$, see [10]. We introduce the matrix ball $B_{q}:=\left\{w \in M_{q}(\mathbb{F}): w^{*} w<I\right\}$, where $A<B$ means for matrices $A, B \in M_{q}(\mathbb{F})$ that $B-A$ is (strictly) positive definite. On $B_{q}$, we define the probability measures

$$
d m_{p}(w)=\frac{1}{\kappa_{p d / 2}} \Delta\left(I-w^{*} w\right)^{p d / 2-\gamma} d w \in M^{1}\left(B_{q}\right),
$$

with $p \in \mathbb{R}, p>2 q-1$. Here $d w$ is the Lebesgue measure on the ball $B_{q}$,

$$
\gamma:=d\left(q-\frac{1}{2}\right)+1
$$

and

$$
\kappa_{p d / 2}=\int_{B_{q}} \Delta\left(I-w^{*} w\right)^{p d / 2-\gamma} d w
$$

According to Theorem 2.4 of [26], the Heckman-Opdam hypergeometric function $F_{B C}(\lambda, k(p), x)$ with $\lambda \in \mathbb{C}^{q}, x \in \mathbb{R}^{q}$ and $k(p)$ as in (1.2) has the following integral representation for $p \in \mathbb{R}$ with $p>2 q-1$ :

$$
F_{B C}(\lambda, k(p), x)=\int_{B_{q} \times U_{0}(q, \mathbb{F})} \Delta_{\left(\lambda-\rho_{p}\right) / 2}\left(g_{x}(u, w)\right) d m_{p}(w) d u,
$$

where $U_{0}(q, \mathbb{F})$ denotes the identity component of $U(q, \mathbb{F})$ and

$$
g_{x}(u, w)=u^{-1}\left(\cosh \underline{x}+w^{*} \sinh \underline{x}\right)(\cosh \underline{x}+\sinh \underline{x} w) u .
$$

It is easily checked that $U_{0}(q, \mathbb{F})$ may be replaced by $U(q, \mathbb{F})$ in the domain of integration. Notice further that $x \mapsto g_{x}(u, w)$ extends to a holomorphic function on $\mathbb{C}^{q}$. As the principal minors $\Delta_{r}(a)$ are polynomial in the entries of $a \in H_{q}(\mathbb{F})$, it follows that $x \mapsto \Delta_{\lambda / 2}\left(g_{x}(u, w)\right)$ extends to a holomorphic function on $\mathbb{C}^{q}$ for each $\lambda \in P_{+}$. In view of relation (1.1), this leads to the following integral representation for the Jacobi polynomials $R_{\lambda}^{p}$ :

Proposition 2.1. Let $p \in \mathbb{R}$ with $p>2 q-1$ and $k(p)=(d(p-q) / 2,(d-1) / 2, d / 2)$ with $d \in\{1,2,4\}$. Then the Jacobi polynomials $R_{\lambda}^{p}, \lambda \in P_{+}$, have the integral representation

$$
R_{\lambda}^{p}(x)=\int_{B_{q} \times U(q, \mathbb{F})} \Delta_{\lambda / 2}\left(g_{i x}(u, w)\right) d m_{p}(w) d u \quad \text { for } \quad x \in A_{0}
$$


with

$$
g_{i x}(u, w)=u^{-1}\left(\cos \underline{x}+w^{*} i \sin \underline{x}\right)(\cos \underline{x}+i \sin \underline{x} w) u .
$$

We next turn to the Bessel functions which will show up in the Mehler-Heine formula. They are given in terms of Bessel functions of Dunkl type which generalize the spherical functions of Cartan motion groups; see [6] and [21] for a general background. We denote by $J_{k}^{B}$ the Bessel function which is associated with the rational Dunkl operators for the root system $B_{q}=$ $\left\{ \pm e_{i}, \pm e_{i} \pm e_{j}: 1 \leq i<j \leq q\right\}$ and multiplicity $k=\left(k_{1}, k_{2}\right)$ corresponding to the roots $\pm e_{i}$ and $\pm e_{i} \pm e_{j}$. We shall be concerned with multiplicities which are connected as follows to the $B C_{q}$ multiplicities $k(p)$ from (1.2):

$$
k=\left(k_{1}, k_{2}\right) \quad \text { with } \quad k_{1}=k(p)_{1}+k(p)_{2}=d(p-q+1) / 2-1 / 2, \quad k_{2}=k(p)_{3}=d / 2 .
$$

For such $k$ on $B_{q}$, we use the notion

$$
\widetilde{\varphi}_{\lambda}^{p}(x):=J_{k}^{B}(x, i \lambda), \quad x \in C, \lambda \in \mathbb{C}^{q} .
$$

It is well-known that for integers $p \geq q$, the $\widetilde{\varphi}_{\lambda}^{p}$ are the spherical functions of the Euclidean symmetric spaces $G_{0} / K$, where $K=S(U(p, \mathbb{F}) \times U(q, \mathbb{F}))$ and $G_{0}=K \ltimes M_{p, q}(\mathbb{F})$ is the Cartan motion group associated with the Grassmannian $\mathcal{G}_{p, q}(\mathbb{F})$. Hereby the double coset space $G_{0} / / K$ is identified with the Weyl chamber $C$ such that $x \in C$ corresponds to the double coset of $\left(I_{p} \times I_{q},\left(I_{p-q}, \underline{x}\right)\right) \in G_{0}$, and in this way, $K$-biinvariant functions on $G_{0}$ may be considered as functions on $C$. Two functions $\widetilde{\varphi}_{\lambda}^{p}$ and $\widetilde{\varphi}_{\mu}^{p}$ coincide if and only if $\lambda$ and $\mu$ are in the same Weyl group orbit. Finally, the bounded spherical functions are exactly those $\widetilde{\varphi}_{\lambda}^{p}$ with $\lambda \in C$. The Bessel functions $\widetilde{\varphi}_{\lambda}^{p}$ with $d=\operatorname{dim}_{\mathbb{F}} \mathbb{R}$ and not necessarily integral parameter $p$ are closely related to Bessel functions on the symmetric cone of positive definite $q \times q$-matrices over $\mathbb{F}$, see Section 4 of [24]. It has been shown there that for $p>2 q-1$, they have a positive product formula which generalizes the product formula in the Cartan motion group cases and leads to a commutative hypergroup structure on the Weyl chamber $C$.

Lemma 2.2. For $p \in \mathbb{R}$ with $p>2 q-1$, the Bessel functions $\widetilde{\varphi}_{\lambda}^{p}$ with $\lambda \in \mathbb{R}^{q}$ have the following integral representation:

$$
\widetilde{\varphi}_{\lambda}^{p}(x)=\int_{B_{q}} \int_{U(q, \mathbb{F})} e^{i \operatorname{Re} \operatorname{tr}(w \underline{x} u \underline{\lambda})} d m_{p}(w) d u .
$$

Proof. This follows readily from equations (3.12) and (4.4) in [24]; see also Proposition 5.3 of $[26]$.

Remark 2.3. There are finitely many geometric cases which are not covered by the range $p \in] 2 q-1, \infty[$, namely the indices $p \in\{q, q+1, \ldots, 2 q-1\}$. In these cases, the Jacobi polynomials $R_{\lambda}^{p}$ and the Bessel functions $\widetilde{\varphi}_{\lambda}^{p}$ both admit interpretations as spherical functions and have an integral representation similar to that above, by the following reasoning: According to Lemma 2.1 of [25], the measure $m_{p} \in M^{1}\left(B_{q}\right)$ with $p \in \mathbb{N}, p \geq 2 q$ is just the pushforward measure of the normalized Haar measure on $U(p, \mathbb{F})$ under the mapping

$$
v \mapsto \sigma_{0}^{*} v \sigma_{0}, \quad \text { with } \quad \sigma_{0}=\left(\begin{array}{c}
I_{q} \\
0_{(p-q) \times q}
\end{array}\right) \in M_{p, q}(\mathbb{F}) .
$$

For $p \in\{q, q+1, \ldots, 2 q-1\}$, we now define the measure $m_{p} \in M^{1}\left(B_{q}\right)$ in the same way as a pushforward measure of the Haar measure on $U(p, \mathbb{F})$. (But in contrast to the case $p \geq 2 q$, it will not have a Lebesgue density in these cases). From the integral representations (3.3) and (4.4) of [24] for the Bessel functions, as well as Theorem 2.1 of [26] and relation (1.1) between Jacobi polynomials and hypergeometric functions, one obtains that the integral representations of Proposition 2.1 and Lemma 2.2 extend to the case $p \in\{q, q+1, \ldots, 2 p-1\}$. 
We shall now compare the integral representations of Proposition 2.1 and Lemma 2.2, which will lead to the following quantitative Mehler-Heine (or Hilb-type) formula.

Theorem 2.4. There exist constants $C_{1}, C_{2}>0$ such that for all $p \in\{q, q+1, \ldots, 2 q-1\} \cup$ ] $2 q-1, \infty\left[\right.$, all $\lambda \in P_{+}$, and $x \in A_{0}$,

$$
\left|R_{\lambda}^{p}(x)-\widetilde{\varphi}_{\lambda}^{p}(x)\right| \leq C_{1} x_{1}^{2} \lambda_{1} e^{C_{2} x_{1}^{2} \lambda_{1}} .
$$

Thus in particular,

$$
\left|R_{n \lambda}^{p}\left(\frac{x}{n}\right)-\widetilde{\varphi}_{\lambda}^{p}(x)\right| \leq \frac{C_{1}}{n} x_{1}^{2} \lambda_{1} e^{C_{2} x_{1}^{2} \lambda_{1} / n} \rightarrow 0 \quad \text { for } \quad n \rightarrow \infty .
$$

Notice that the estimate of Theorem 2.4 is uniform in $p$, a fact which was to our knowledge so far not even noticed in the rank-one case. We conjecture that the statement of this theorem remains correct for $p \in[q, \infty[$.

Proof. We only consider the case $p>2 q-1$ where the proof is based on Proposition 2.1 and Lemma 2.2. By the previous remark, the cases $p=q, q+1, \ldots, 2 q-1$ can be treated in the same way. Notice that it suffices to check uniformity in $p$ for $p>2 q-1$.

We substitute $w \mapsto u^{*} w^{*}$ in the integral (2.2) and obtain

$$
\widetilde{\varphi}_{\lambda}^{p}(x)=\int_{B_{q} \times U(q, \mathbb{F})} e^{i \operatorname{Re} \operatorname{tr}\left(u^{*} w^{*} \underline{x} u \underline{\lambda}\right)} d m_{p}(w) d u .
$$

Denoting the trace of the upper left $(r \times r)$-block of a $(q \times q)$-matrix by $\operatorname{tr}_{r}$, we have

$$
\begin{aligned}
\operatorname{Re} \operatorname{tr}\left(u^{*} w^{*} \underline{x} u \underline{\lambda}\right) & =\frac{1}{2} \sum_{r=1}^{q}\left(u^{*}\left((\underline{x} w)^{*}+\underline{x} w\right) u\right)_{r r} \lambda_{r} \\
& =\sum_{r=1}^{q}\left[\operatorname{tr}_{r}\left(u^{*}\left((\underline{x} w)^{*}+\underline{x} w\right) u\right)-\operatorname{tr}_{r-1}\left(u^{*}\left((\underline{x} w)^{*}+\underline{x} w\right) u\right)\right] \lambda_{r} / 2 \\
& =\sum_{r=1}^{q} \operatorname{tr}_{r}\left(u^{*}\left((\underline{x} w)^{*}+\underline{x} w\right) u\right)\left(\lambda_{r}-\lambda_{r+1}\right) / 2
\end{aligned}
$$

with $\lambda_{q+1}:=0$. Hence

$$
\widetilde{\varphi}_{\lambda}^{p}(x)=\int_{B_{q} \times U(q, \mathbb{F})} \prod_{r=1}^{q} e^{i \operatorname{tr}_{r}\left(u^{*}\left((x w)^{*}+x w\right) u\right)\left(\lambda_{r}-\lambda_{r+1}\right) / 2} d m_{p}(w) d u .
$$

Furthermore, by Proposition 2.1,

$$
R_{\lambda}^{p}(x)=\int_{B_{q} \times U(q, \mathbb{F})} \prod_{r=1}^{q} \Delta_{r}\left(g_{i x}(u, w)\right)^{\left(\lambda_{r}-\lambda_{r+1}\right) / 2} d m_{p}(w) d u .
$$

Telescope summation yields the well-known estimate

$$
\left|\prod_{r=1}^{q} a_{r}-\prod_{r=1}^{q} b_{r}\right| \leq\left(\max \left(\left|a_{1}\right|, \ldots,\left|a_{q}\right|,\left|b_{1}\right|, \ldots,\left|b_{q}\right|\right)\right)^{q-1} \sum_{r=1}^{q}\left|a_{r}-b_{r}\right|
$$

for $a_{1}, \ldots, a_{q}, b_{1}, \ldots, b_{q} \in \mathbb{C}$. We thus obtain

$$
\left|R_{\lambda}^{p}(x)-\widetilde{\varphi}_{\lambda}^{p}(t)\right| \leq \sum_{r=1}^{q} \int_{B_{q} \times U(q, \mathbb{F})} M(x, u, w, \lambda)
$$




$$
\times\left|\Delta_{r}\left(g_{x}(u, w)\right)^{\left(\lambda_{r}-\lambda_{r+1}\right) / 2}-e^{i \operatorname{tr}_{r}\left(u^{*}\left((\underline{t} w)^{*}+\underline{t} w\right) u\right)\left(\lambda_{r}-\lambda_{r+1}\right) / 2}\right| d m_{p}(w) d u
$$

with

$$
M(x, u, w, \lambda):=\max \left(1, \max _{r=1, \ldots, q}\left|\Delta_{r}\left(g_{x}(u, w)\right)^{\left(\lambda_{r}-\lambda_{r+1}\right) / 2}\right|^{q-1}\right) .
$$

We now investigate $\Delta_{r}\left(g_{i x}(u, w)\right)^{\left(\lambda_{r}-\lambda_{r+1}\right) / 2}$ more closely. As $x, u, w$ run through compacta, we obtain that uniformly in $x, u, w$,

$$
\begin{aligned}
g_{i x}(u, w) & =u^{-1}\left(\cos \underline{x}+w^{*} i \sin \underline{x}\right)(\cos \underline{x}+i \sin \underline{x} w) u \\
& =u^{-1}\left(I_{q}+w^{*} i \underline{x}+O\left(x^{2}\right)\right)\left(I_{q}+i \underline{x} w+O\left(x^{2}\right)\right) u \\
& =I_{q}+u^{-1}\left(i \underline{x} w+w^{*} i \underline{x}\right) u+O\left(x^{2}\right),
\end{aligned}
$$

and thus

$$
\Delta_{r}\left(g_{x}(u, w)\right)=1+\operatorname{tr}_{r}\left(u^{-1}\left(i \underline{x} w+w^{*} i \underline{x}\right) u\right)+O\left(x^{2}\right) .
$$

Using the power series for $\ln (1+z)$, we further have

$$
\begin{aligned}
\Delta_{r}\left(g_{i x}(u, w)\right)^{\left(\lambda_{r}-\lambda_{r+1}\right) / 2} & =\exp \left[\frac{1}{2}\left(\lambda_{r}-\lambda_{r+1}\right) \ln \left(1+\operatorname{tr}_{r}\left(u^{-1}\left(i \underline{x} w+w^{*} i \underline{x}\right) u\right)+O\left(x^{2}\right)\right)\right] \\
& =\exp \left[\frac{1}{2}\left(\lambda_{r}-\lambda_{r+1}\right) \operatorname{tr}_{r}\left(u^{-1}\left(\underline{x} w+w^{*} i \underline{x}\right) u\right)+O\left(x^{2}\right)\left(\lambda_{r}-\lambda_{r+1}\right)\right] .
\end{aligned}
$$

Notice that $y:=u^{-1}\left(i \underline{x} w+w^{*} i \underline{x}\right) u$ is skew-Hermitian, that is $y^{*}=-y$. Therefore $\overline{\operatorname{tr}_{r}(y)}=$ $-\operatorname{tr}_{r}(y)$, which implies that $\operatorname{Re}\left(\operatorname{tr}_{r}(y)\right)=0$. It follows that

$$
\left|\Delta_{r}\left(g_{i x}(u, w)\right)^{\left(\lambda_{r}-\lambda_{r+1}\right) / 2}\right|=\exp \left[\frac{1}{2}\left(\lambda_{r}-\lambda_{r+1}\right) \operatorname{Re}\left(\operatorname{tr}_{r}(y)+O\left(x^{2}\right)\right)\right]=e^{\left(\lambda_{r}-\lambda_{r+1}\right) O\left(x^{2}\right)} .
$$

Note that these considerations apply for all fields $\mathbb{F}=\mathbb{R}, \mathbb{C}, \mathbb{H}$. It follows that there exists a constant $C_{3}>0$ (independent of $x, u, w, \lambda$ ) such that

$$
M(x, u, w, \lambda) \leq e^{C_{3} x_{1}^{2} \lambda_{1}} \quad \text { for all } \quad x \in A_{0}, \lambda \in P_{+}, u \in U(q, \mathbb{F}), w \in B_{q} .
$$

From this inequality we obtain by the mean value theorem that for all $x \in A_{0}$ and $\lambda \in P_{+}$,

$$
\begin{aligned}
& \left|\Delta_{r}\left(g_{x}(u, w)\right)^{\left(\lambda_{r}-\lambda_{r+1}\right) / 2}-e^{i \operatorname{tr}_{r}\left(u^{*}\left((\underline{x} w)^{*}+\underline{x} w\right) u\right)\left(\lambda_{r}-\lambda_{r+1}\right) / 2}\right| \\
& \quad \leq e^{C_{3} x_{1}^{2} \lambda_{1}}-1 \leq C_{3} x_{1}^{2} \lambda_{1} e^{C_{3} x_{1}^{2} \lambda_{1}} .
\end{aligned}
$$

These estimates together with (2.3) imply the assertion.

Example 2.5 (the rank one case). For $q=1$ the Jacobi polynomials $R_{\lambda}^{p}$ associated with root system $B C_{1}=\left\{ \pm e_{1}, \pm 2 e_{1}\right\}$ are classical one-variable Jacobi polynomials in trigonometric parametrization. For integers $p$, the associated Grassmannians are the projective spaces $P_{p}(\mathbb{F})$. For the details, recall that the classical Jacobi polynomials $R_{n}^{(\alpha, \beta)}$ with the normalization $R_{n}^{(\alpha, \beta)}(1)=1$ are given by

$$
R_{n}^{(\alpha, \beta)}(x)={ }_{2} F_{1}(\alpha+\beta+n+1,-n ; \alpha+1 ;(1-x) / 2),
$$

where $n \in \mathbb{Z}_{+}, \alpha, \beta>-1$. It is easily derived from the example on p. 17 of [22] that

$$
R_{\lambda}^{p}(x)=R_{\lambda / 2}^{(\alpha, \beta)}(\cos 2 x)
$$

for $\lambda \in 2 \mathbb{Z}_{+}$, with

$$
\alpha=(d p-2) / 2, \quad \beta=(d-2) / 2 ;
$$


see also [23, Section 5]. In the rank one case, the $U(1, \mathbb{F})$ integral in representation (2.1) cancels by invariance of $\Delta$ under unitary conjugation. Thus (2.1) reduces to

$$
R_{\lambda}^{p}(x)=\frac{1}{\kappa_{p d / 2}} \int_{B_{1}}((\cos x+\bar{w} i \sin x)(\cos x+i \sin x w))^{\lambda / 2}\left(1-|w|^{2}\right)^{d(p-1) / 2-1} d w
$$

for $\lambda \in \mathbb{Z}_{+}, p>1$. In particular, if $\mathbb{F}=\mathbb{R}$, then $d=1$ and $B_{1}=[-1,1]$. Thus

$$
R_{n}^{(p / 2-1,-1 / 2)}(\cos 2 x)=\frac{1}{\kappa_{p / 2}} \int_{-1}^{1}(\cos x+i t \sin x)^{2 n}\left(1-t^{2}\right)^{(p-3) / 2} d t .
$$

If $\mathbb{F}=\mathbb{C}$, then $d=2$ and $B_{1}=\{z \in \mathbb{C}:|z| \leq 1\}$. Using polar coordinates $z=t e^{i \theta}$, one obtains

$$
R_{n}^{(p-1,0)}(\cos 2 x)=\frac{1}{\kappa_{p}} \int_{-1}^{1} \int_{0}^{\pi}\left(\left(\cos x+i t e^{i \theta} \sin x\right)\left(\cos x+i t e^{-i \theta} \sin x\right)\right)^{2 n}\left(1-t^{2}\right)^{p-2} t d t d \theta .
$$

The quaternionic case can be treated in a similar way. These formulas are just special cases of a well-known Laplace-type integral representation for Jacobi polynomials with general indices $\alpha \geq \beta \geq-1 / 2$; see, e.g., [20, Section 18.10].

Let us finally mention that the Mehler-Heine formula 2.4 corresponds to [27, Theorem 8.21.12] and that in the case of rank two $(q=2)$, the Jacobi polynomials of type BC were first studied by Koornwinder [16, 17].

\section{Random walks on the dual of a compact Grassmannian and on $P_{+}$}

Recall that for integers $p \geq q$ the functions $\varphi_{\lambda}:=\varphi_{\lambda}^{p}, \lambda \in P_{+}$form the spherical functions of the compact Grassmannians $G / K=\mathcal{G}_{p, q}(\mathbb{F})$. As functions on $G$, they are positive-definite. In other words, the Jacobi polynomials $\left(R_{\lambda}^{p}\right)_{\lambda \in P_{+}}$are just the hypergroup characters of the compact double coset hypergroups $G / / K \cong A_{0}$. We now recapitulate the associated dual hypergroup structures on $P_{+}$.

\subsection{Dual hypergroup structures on $\boldsymbol{P}_{+}$}

As mentioned in the introduction, there is a one-to-one correspondence between the set of (positive definite) spherical functions of $G / K$, which is parametrized by $P_{+}$, and the spherical unitary dual of $G / K$, i.e., the set $\widehat{G}_{K}$ of all equivalence classes of irreducible unitary representations $(\pi, H)$ of $G$ whose restriction to $K$ contains the trivial representation with multiplicity one. Here a representation $(\pi, H) \in \widehat{G}_{K}$ and its spherical function $\varphi_{\pi}$ are related by

$$
\varphi_{\pi}(x)=\langle u, \pi(x) u\rangle \quad \text { for } \quad x \in G
$$

with some $K$-invariant vector $u \in H$ with $\|u\|=1$, which is determined uniquely up to a complex constant of absolute value 1 .

Now consider $\lambda, \mu \in P_{+}$with associated spherical functions $\varphi_{\lambda}, \varphi_{\mu}$ and the corresponding representations $\left(\pi_{\lambda}, H_{\lambda}\right),\left(\pi_{\mu}, H_{\mu}\right) \in \widehat{G}_{K}$ with $K$-invariant vectors $u_{\lambda}, u_{\mu}$. The tensor product $\left(\pi_{\lambda} \otimes \pi_{\mu}, H_{\lambda} \otimes H_{\mu}\right)$ is a finite-dimensional unitary representation of $G$ which decomposes into a finite orthogonal sum

$$
\left(\oplus_{k} \tau_{k}=\pi_{\lambda} \otimes \pi_{\mu}, \oplus_{k} \widetilde{H}_{k}=H_{\lambda} \otimes H_{\mu}\right)
$$


of irreducible unitary representations $\left(\tau_{k}, \widetilde{H}_{k}\right)$ where some of them may appear several times. Consider the orthogonal projections $p_{k}: H_{\lambda} \otimes H_{\mu} \rightarrow \widetilde{H}_{k}$. Then the vectors $p_{k}\left(u_{\lambda} \otimes u_{\mu}\right) \in \widetilde{H}_{k}$ are $K$-invariant, and for $p_{k}\left(u_{\lambda} \otimes u_{\mu}\right) \neq 0$, we obtain $\left(\tau_{k}, \widetilde{H}_{k}\right) \in \widehat{G}_{K}$, i.e., $\left(\tau_{k}, \widetilde{H}_{k}\right)$ is equal to some $\left(\pi_{\tau}, H_{\tau}\right), \tau \in P_{+}$. Moreover, for $g \in G$,

$$
\begin{aligned}
\varphi_{\lambda}(g) \varphi_{\mu}(g) & =\left\langle u_{\lambda} \otimes u_{\mu},\left(\pi_{\lambda} \otimes \pi_{\mu}\right)(g) u_{\lambda} \otimes u_{\mu}\right\rangle \\
& =\sum_{k}\left\langle p_{k}\left(u_{\lambda} \otimes u_{\mu}\right), \tau_{k}(g) p_{k}\left(u_{\lambda} \otimes u_{\mu}\right)\right\rangle=\sum_{k}\left\|p_{k}\left(u_{\lambda} \otimes u_{\mu}\right)\right\|^{2} \varphi_{\tau_{k}}(g)
\end{aligned}
$$

with $\sum_{k}\left\|p_{k}\left(u_{\lambda} \otimes u_{\mu}\right)\right\|^{2}=1$. For $\lambda, \mu, \tau \in P_{+}$we now define $c_{\lambda, \mu, \tau} \geq 0$ as $\left\|p_{k}\left(u_{\lambda} \otimes u_{\mu}\right)\right\|^{2}$, whenever $\left(\tau_{k}, \widetilde{H}_{k}\right)=\left(\pi_{\tau}, H_{\tau}\right)$ appears above with a positive part, and 0 otherwise. As $\varphi_{\lambda}(g) \in \mathbb{R}$ for all $\lambda, g$ in our case, these nonnegative linearization coefficients also satisfy

$$
c_{\lambda, \mu, \tau}=\operatorname{dim} H_{\tau} \int_{G} \varphi_{\lambda}(g) \varphi_{\mu}(g) \varphi_{\tau}(g) d g .
$$

For $\lambda, \mu \in P_{+}$we define the probability measure

$$
\delta_{\lambda} *_{d, p} \delta_{\mu}:=\sum_{\tau \in P_{+}} c_{\lambda, \mu, \tau} \delta_{\tau} \in M^{1}\left(P_{+}\right)
$$

with finite support. By its very construction, this convolution can be extended uniquely in a weakly continuous, bilinear way to a probability preserving, commutative, and associative convolution on the Banach space $M_{b}\left(P_{+}\right)$of all bounded, signed measures on $P_{+}$. Moreover, as all spherical functions are $\mathbb{R}$-valued in our specific examples, the contragredient representation of any element in $\widehat{G}_{K}$ is the same representation, i.e., the canonical involution * $^{*}$ on $P_{+} \cong \widehat{G}_{K}$ is the identity. In summary, $\left(M_{b}\left(P_{+}\right), *_{d, p}\right)$ is a commutative Banach-*-algebra with the complex conjugation $\mu^{*}(A):=\overline{\mu(A)}$ as involution. Moreover, $\left(P_{+}, *_{d, p}\right)$ becomes a so-called Hermitian hypergroup in the sense of Dunkl, Jewett and Spector; see [3, 7, 15].

The Haar measure on this hypergroup, which is unique up to a multiplicative constant, is the positive measure $\omega=\sum_{\lambda \in P_{+}} h(\lambda) \delta_{\lambda}$ with

$$
h(\lambda)=c_{\lambda, \lambda, 0}^{-1}=\int_{G} \varphi_{\lambda}^{2}(g) d g=\frac{1}{\operatorname{dim} H_{\lambda}},
$$

where the first two equations follow from general hypergroup theory (see [15]) and the last one from the theory of Gelfand pairs (see, e.g., [9]).

The coefficients $c_{\lambda, \mu, \tau}$ of the convolution $*_{d, p}$ on $P_{+}$are related to the unique product linearization

$$
R_{\lambda}^{p} R_{\mu}^{p}=\sum_{\tau \in P_{+}} c_{\lambda, \mu, \tau} R_{\tau}^{p}
$$

of the Jacobi polynomials $R_{\lambda}^{p}$. It is clear by our construction that for integers $p \geq q$, all $c_{\lambda, \mu, \tau}$ are nonnegative with $\sum_{\tau \in P_{+}} c_{\lambda, \mu, \tau}=1$.

Clearly, as $R_{\lambda}^{p}(0)=1$ for all $\lambda$, the normalization also holds for all real $p \in[q, \infty[$. We conjecture that actually all $c_{\lambda, \mu, \tau}$ are nonnegative for all $p \in[q, \infty[$ or at least for all $p \in$ $[2 q-1, \infty[$.

Suppose that for fixed $p \in\left[q, \infty\right.$ [ the linearization coefficients $c_{\lambda, \mu, \tau}$ are all nonnegative. Then equation (3.1) defines a commutative discrete hypergroup structure $\left(P_{+}, *_{d, p}\right)$ with the convolution

$$
\delta_{\lambda} *_{d, p} \delta_{\mu}:=\sum_{\tau \in P_{+}} c_{\lambda, \mu, \tau} \delta_{\tau} \in M^{1}\left(P_{+}\right)
$$


of point measures. For instance, in the rank one case of Example 2.5 the linearization coefficients $c_{\lambda, \mu, \tau}$ are explicitly known and nonnegative for all $p \geq 1$ as the product linearization coefficients of the associated one-dimensional Jacobi polynomials.

\subsection{Random walks on $\boldsymbol{P}_{+}$}

We next introduce certain random walks on $P_{+}$, i.e., time-homogeneous Markov chains on $P_{+}$ whose transition probabilities are given in terms of the product linearizations coefficients $c_{\lambda, \mu, \tau}$ for some fixed $p \in[q, \infty[$. This concept even works, under a suitable restriction, in the case where some of the $c_{\lambda, \mu, \tau}$ are negative. To describe the restriction, we fix $p \in[q, \infty[$ and define the set

$$
P_{+}^{p}:=\left\{\lambda \in P_{+}: c_{\lambda, \mu, \tau} \geq 0 \text { for all } \mu, \tau \in P_{+}\right\}
$$

as well as

$$
M_{p}^{1}\left(P_{+}\right):=\left\{\nu \in M^{1}\left(P_{+}\right): \operatorname{supp} \nu \subset P_{+}^{p}\right\} .
$$

We shall call the probability measures $\nu \in M_{p}^{1}\left(P_{+}\right)$admissible.

Clearly, for integers $p \geq q$, as well as for $q=1$ we have $M_{p}^{1}\left(P_{+}\right)=M^{1}\left(P_{+}\right)$. Unfortunately, it seems difficult to find further examples. To illustrate the problem, consider the measure $\delta_{(1,0, \ldots, 0)} \in M^{1}\left(P_{+}\right)$for $q \geq 2$ and $p \in[q, \infty$. The explicit Pieri-type formula (6.4) of [28] then leads to a concrete product linearization formula for $R_{\lambda}^{p} R_{(1,0, \ldots, 0)}^{p}$. It can be easily derived from [28] that for all $q \geq 2, p \in\left[q, \infty\right.$ [, and all $\lambda \neq \mu$, we have $c_{\lambda,(1,0, \ldots, 0), \mu} \geq 0$ as desired. However, we are so far not able to check from [28] that $c_{\lambda,(1,0, \ldots, 0), \lambda} \geq 0$ holds, which would be necessary for $\delta_{(1,0, \ldots, 0)} \in M_{p}^{1}\left(P_{+}\right)$.

As before, we fix $d=1,2,4$ and $p \in[q, \infty[$. We also fix an admissible probability measure $\nu=\sum_{\mu} p_{\mu} \delta_{\mu} \in M_{p}^{1}\left(P_{+}\right)$and consider a time-homogeneous Markov chain $\left(S_{n}^{d, p}\right)_{n \geq 0}$ in discrete time on $P_{+}$starting at time 0 in the origin $0 \in P_{+}$and with transition probability

$$
P\left(S_{n+1}^{d, p}=\tau \mid S_{n}^{d, p}=\lambda\right)=\left(\nu *_{d, p} \delta_{\lambda}\right)(\{\tau\}), \quad \lambda, \tau \in P_{+}, \quad n \in \mathbb{N},
$$

where

$$
\nu *_{d, p} \delta_{\lambda}:=\sum_{\tau}\left(\sum_{\mu} p_{\mu} c_{\lambda, \mu, \tau}\right) \delta_{\tau} \in M^{1}\left(P_{+}\right) .
$$

Such Markov-chains are called random walks on $\left(P_{+}, *_{d, p}\right)$ associated with $\nu$. It is well-known and can be easily checked by induction that for all $n$ the $n$-fold convolution power $\nu^{(n)}:=$ $\nu *_{d, p} \cdots *_{d, p} \nu \in M^{1}\left(P_{+}\right)$exists, and that $\nu^{(n)}$ is just the distribution $P_{S_{n}^{d, p}}$ of $S_{n}^{d, p}$.

In view of the Central Limit Theorem 1.1, we give an interpretation of these convolution powers $\delta_{\lambda}^{(n)}$ for integers $p \geq q$ and $\lambda \in P_{+}$with $\lambda \neq 0$ in terms of representation theory. We expect that this result is well-known, but we do not know an explicit proof in the literature.

Lemma 3.1. Let $\left(\pi_{\lambda}, H_{\lambda}\right)$ be the non-trivial irreducible unitary representation of $G$ associated with $\lambda \in P_{+}, \lambda \neq 0$ and with a $K$-invariant vector $u_{\lambda} \in H_{\lambda}$ with $\left\|u_{\lambda}\right\|=1$. For each $n \in \mathbb{N}$, decompose the $n$-th tensor power $\left(\pi_{\lambda}^{\otimes, n}, H_{\lambda}^{\otimes, n}\right)$ into its irreducible components

$$
\left(\pi_{\lambda}^{\otimes, n}, H_{\lambda}^{\otimes, n}\right)=\left(\bigoplus_{\tau_{n}} \pi_{\tau_{n}}, \bigoplus_{\tau_{n}} H_{\tau_{n}}\right)
$$

and consider the orthogonal projections $p_{\tau_{n}}: H_{\lambda}^{\otimes, n} \rightarrow H_{\tau_{n}}$. Then for all $n \in \mathbb{N}$,

$$
\delta_{\lambda}^{(n)}=\sum_{\tau_{n}}\left\|p_{\tau_{n}}\left(u_{\lambda}^{\otimes, n}\right)\right\|^{2} \delta_{\tau_{n}} .
$$


Proof. We proceed by induction. In fact, the case $n=1$ is trivial. For $n \rightarrow n+1$, we start with (3.2) and the associated orthogonal projections $p_{\tau_{n}}: H_{\lambda}^{\otimes, n} \rightarrow H_{\tau_{n}}$. We now decompose the products $\pi_{\tau_{n}} \otimes \pi_{\lambda}$ and obtain

$$
\begin{aligned}
\left(\pi_{\lambda}^{\otimes, n+1}, H_{\lambda}^{\otimes, n+1}\right) & =\left(\bigoplus_{\tau_{n}}\left(\pi_{\tau_{n}} \otimes \pi_{\lambda}\right), \bigoplus_{\tau_{n}}\left(H_{\tau_{n}} \otimes H_{\lambda}\right)\right) \\
& =\left(\bigoplus_{\tau_{n}}\left(\bigoplus_{\mu_{k, n}} \pi_{\mu_{k, n}}\right), \bigoplus_{\tau_{n}}\left(\bigoplus_{\mu_{k, n}} H_{\mu_{k, n}}\right)\right) .
\end{aligned}
$$

Notice that here the sum $\bigoplus_{\tau_{n}} \bigoplus_{\mu_{k, n}}$ corresponds to the sum $\bigoplus_{\tau_{n+1}}$ of the lemma with $n+1$ instead of $n$. We now consider the orthogonal projections $p_{\mu_{k, n}}: H_{\lambda}^{\otimes, n+1} \rightarrow H_{\mu_{k, n}}$. Then

$$
p_{\tau_{n}}\left(u_{\lambda}^{\otimes, n}\right)=c\left\|p_{\tau_{n}}\left(u_{\lambda}^{\otimes, n}\right)\right\| u_{\tau_{n}},
$$

where $|c|=1$, and thus

$$
\left\|p_{\mu_{k, n}}\left(u_{\lambda}^{\otimes, n+1}\right)\right\|^{2}=\left\|p_{\mu_{k, n}}\left(p_{\tau_{n}}\left(u_{\lambda}^{\otimes, n}\right) \otimes u_{\lambda}\right)\right\|^{2}=\left\|p_{\tau_{n}}\left(u_{\lambda}^{\otimes, n}\right)\right\|^{2}\left\|p_{\mu_{k, n}}\left(u_{\tau_{n}} \otimes u_{\lambda}\right)\right\|^{2} .
$$

This fact, the assumption of our induction and the definition of the convolution now readily imply the assertion of the lemma for $n+1$.

We shall prove below that under a natural moment condition on a probability measure $\nu \in$ $M^{1}\left(P_{+}\right)$, the $C$-valued random variables $\frac{1}{\sqrt{n}} S_{n}^{d, p}$ converge in distribution for $n \rightarrow \infty$. In order to identify the limit distribution $\mu=\mu(d, p, \nu) \in M^{1}(C)$ in this central limit theorem, we need some further preparations.

\subsection{Bessel convolutions on $C$ and Laguerre ensembles}

As described in Section 2, the Bessel functions $\widetilde{\varphi}_{\lambda}^{p}$ with $\lambda \in C$ make up the set of bounded spherical functions of the Euclidean symmetric space $G_{0} / K$ with $K=U(p, \mathbb{F}) \times U(q, \mathbb{F})$ and $G_{0}=K \ltimes M_{p, q}(\mathbb{F})$. Thus by the notation of [3] and [15], the chamber $C \cong G_{0} / / K$ with the associated double coset convolution $\bullet_{d, p}$ is a commutative double coset hypergroup with the functions $\widetilde{\varphi}_{\lambda}^{p}$ as (bounded) hypergroup characters. We now introduce the probability measures

$$
d \rho_{d, p}(x):=c_{d, p}^{-1} \prod_{j=1}^{q} x_{j}^{d(p-q+1)-1} \prod_{1 \leq i<j \leq q}\left(x_{i}^{2}-x_{j}^{2}\right)^{d} e^{-\left(x_{1}^{2}+\cdots+x_{q}^{2}\right) / 2} d x
$$

on the Weyl chamber $C$, with the normalization constant

$$
c_{d, p}=\int_{C} \prod_{j=1}^{q} x_{j}^{d(p-q+1)-1} \prod_{1 \leq i<j \leq q}\left(x_{i}^{2}-x_{j}^{2}\right)^{d} e^{-\left(x_{1}^{2}+\cdots+x_{q}^{2}\right) / 2} d x .
$$

The measure $\rho_{d, p} \in M^{1}(C)$ is well-known in the random matrix theory of so-called Laguerreor $\beta$-ensembles as it is the distribution of the singular values of a $M_{p, q}(\mathbb{F})$-valued random variable for which the real and imaginary parts of all entries are i.i.d. and standard normally distributed. This fact is well-known; it can also be derived from the Haar measure of the double coset hypergroups $\left(C, \bullet_{d, p}\right)$ in [24, Section 4.1]. Moreover, having this group theoretic interpretation in mind, one easily obtains the following well-known relation from the Fourier transform of a multivariate standard normal distribution:

$$
\int_{C} \widetilde{\varphi}_{\lambda}^{p}(x) d \rho_{d, p}(x)=e^{-\left(\lambda_{1}^{2}+\cdots+\lambda_{1}^{2}\right) / 2} \quad \text { for } \quad \lambda \in C,
$$

see [10, Proposition XV.2.1] or [30]. This identification of the spherical Fourier-transform of $\rho_{d, p}$ will be essential in the following for the central limit theorem. 


\subsection{A central limit theorem for random walks on $\boldsymbol{P}_{+}$}

The probability measure $\rho_{d, p}$ appears in the CLT below as limit up to some scaling parameter $\sigma^{2}=\sigma^{2}(\nu, p, d)$, which admits an interpretation as a variance parameter. For the description of $\sigma^{2}$, we need the so-called moment functions on $\left(P_{+}, *_{d, p}\right)$ up to order two. For the general theory of such moment functions and their applications to limit theorems for random walks on hypergroups we refer to [3, Chapter 7] and [32], and the references there. The moment functions are characterized by additive functional equations similar to the multiplicative ones for hypergroup characters. They are usually defined in terms of (partial) derivatives with respect to the spectral variables at the identity character. In our examples, the identity corresponds to $x=0 \in C$. This motivates the following definition.

Definition 3.2. Let $p \in[q, \infty$ [ be fixed. For $k, l=1, \ldots, q$ we define the moment functions $m_{k}, m_{k, l}: P_{+} \rightarrow \mathbb{R}$ of the first and second order by

$$
m_{k}(\lambda):=\left.i \frac{\partial}{\partial x_{k}} R_{\lambda}(x)\right|_{x=0} \quad \text { and } \quad m_{k, l}(\lambda):=-\left.\frac{\partial^{2}}{\partial x_{k} \partial x_{l}} R_{\lambda}(x)\right|_{x=0} .
$$

Let us collect some properties of these moment functions.

\section{Lemma 3.3.}

(1) For all $k, l=1, \ldots, q$ with $k \neq l$ and all $\lambda \in P_{+}, m_{k}(\lambda)=m_{k, l}(\lambda)=0$.

(2) The functions $m_{k, k}$ are independent of $k=1, \ldots, q$, and the function $m:=m_{1,1}: P_{+} \rightarrow \mathbb{R}$ is a second order polynomial of the form

$$
m(\lambda)=\frac{1}{4} \sum_{r, s=1}^{q} a_{r, s}\left(\lambda_{r}-\lambda_{r+1}\right)\left(\lambda_{s}-\lambda_{s+1}\right)+\frac{1}{2} \sum_{r=1}^{q} b_{r}\left(\lambda_{r}-\lambda_{r+1}\right)
$$

with suitable coefficients $a_{r, s}, b_{r}$. In particular, $m(0)=0$.

(3) For all $\lambda, \tau \in P_{+}, \int_{P_{+}} m d\left(\delta_{\lambda} *_{d, p} \delta_{\tau}\right)=m(\lambda)+m(\tau)$.

Proof. The Jacobi polynomials $R_{\lambda}(x)$ are invariant under the Weyl group of type B acting in the variable $x$. In particular, $R_{\lambda}\left(x_{1}, \ldots, x_{q}\right)$ is even in each $x_{i}$, and this gives part (1). Moreover, as $R_{\lambda}\left(x_{1}, \ldots, x_{q}\right)$ is invariant under permutations of the $x_{i}$, the function $m_{k, k}$ is independent of $k$. We now study $m:=m_{1,1}$ more closely. We start with the case $p>2 q-1$. In this case we obtain from the integral representation (2.1) that

$$
m(\lambda)=-\left.\int_{B_{q} \times U(q, \mathbb{F})} \frac{\partial^{2}}{\partial x_{1}^{2}}\left(\Delta_{\lambda / 2}\left(g_{i x}(u, w)\right)\right)\right|_{x=0} d m_{p}(w) d u
$$

with the power function

$$
\Delta_{\lambda / 2}\left(g_{i x}(u, w)\right)=\prod_{r=1}^{q} \Delta_{r}\left(g_{i x}(u, w)\right)^{\left(\lambda_{r}-\lambda_{r+1}\right) / 2} \quad \text { with } \quad \lambda_{q+1}=0 .
$$

A short calculation, using that $\Delta_{\lambda / 2}\left(g_{0}(u, w)\right)=1$, gives

$$
\begin{aligned}
\left.\frac{\partial^{2}}{\partial x_{1}^{2}} \Delta_{\lambda / 2}\left(g_{i x}(u, w)\right)\right|_{x=0}= & \frac{1}{4}\left(\left.\sum_{r=1}^{q} \frac{\partial}{\partial x_{1}}\left(\Delta_{r}\left(g_{i x}(u, w)\right)\right)\right|_{x=0}\left(\lambda_{r}-\lambda_{r+1}\right)\right)^{2} \\
& +\left.\frac{1}{2} \sum_{r=1}^{q} \frac{\partial^{2}}{\partial x_{1}^{2}}\left(\ln \Delta_{r}\left(g_{i x}(u, w)\right)\right)\right|_{x=0}\left(\lambda_{r}-\lambda_{r+1}\right) .
\end{aligned}
$$


Formulas (3.4) and (3.5) now imply that $m$ is a second order polynomial as claimed, with linear terms

$$
b_{r}=\left.\int_{B_{q} \times U(q, \mathbb{F})} \frac{\partial^{2}}{\partial x_{1}^{2}}\left(\ln \Delta_{r}\left(g_{i x}(u, w)\right)\right)\right|_{x=0} d m_{p}(w) d u .
$$

The coefficients $a_{r, s}$ are obtained from the Taylor expansion (2.4) of $\Delta_{r}\left(g_{i x}(u, w)\right)$. They are given by

$$
a_{r, s}:=\int_{B_{q} \times U(q, \mathbb{F})} \operatorname{tr}_{r}\left(u^{*}\left(w^{*} P_{1}+P_{1} w\right) u\right) \operatorname{tr}_{s}\left(u^{*}\left(w^{*} P_{1}+P_{1} w\right) u\right) d m_{p}(w) d u
$$

with the diagonal matrix $P_{1}:=\operatorname{diag}(1,0, \ldots, 0) \in M_{q}(\mathbb{F})$. This proves that $m$ is a second order polynomial for $p>2 q-1$. The case $p \geq q$ follows by analytic continuation.

Finally, for the proof of part (3) we observe that for $\lambda, \tau \in P_{+}$,

$$
\begin{aligned}
\int_{P_{+}} m d\left(\delta_{\lambda} *_{d, p} \delta_{\tau}\right) & =-\left.\int_{P_{+}} \frac{\partial^{2}}{\partial x_{1}^{2}} R_{\kappa}(x)\right|_{x=0} d\left(\delta_{\lambda} *_{d, p} \delta_{\tau}\right)(\kappa) \\
& =-\left.\frac{\partial^{2}}{\partial x_{1}^{2}}\left(\int_{P_{+}} R_{\kappa}(x) d\left(\delta_{\lambda} *_{d, p} \delta_{\tau}\right)\right)\right|_{x=0} \\
& =-\left.\frac{\partial^{2}}{\partial x_{1}^{2}}\left(R_{\lambda}(x) R_{\tau}(x)\right)\right|_{x=0}=m(\lambda)+m(\tau) .
\end{aligned}
$$

Notice that the last equality follows from part $(1)$ and $R_{\lambda}(0)=1$.

Example 3.4 (the rank one case). For $q=1$, the moment function $m$ is given by

$$
m(\lambda)=\frac{\lambda(\lambda+d p+d-2)}{d p}, \quad \lambda \in 2 \mathbb{Z}^{+} .
$$

In fact, this can be easily derived from the definition of $m$ and the explicit formulas for the classical Jacobi polynomials in (2.5) and (2.6). Moreover, it can be also derived from the proof of part (2) of the preceding lemma and a direct elementary computation of $a_{1,1}$ and $b_{1}$ for $q=1$ there.

We shall need the following variant of Lemma 3.3(2) on the growth of $m$ for $p \in[q, \infty[$ :

Lemma 3.5. For all $x \in \mathbb{R}^{q}, \lambda \in P_{+}$and $k, l=1, \ldots, q$,

$$
\left|\frac{\partial}{\partial x_{k}} R_{\lambda}^{p}(x)\right| \leq \lambda_{1} \quad \text { and } \quad\left|\frac{\partial^{2}}{\partial x_{k} \partial x_{l}} R_{\lambda}^{p}(x)\right| \leq \lambda_{1}^{2} .
$$

In particular, $m(\lambda) \leq \lambda_{1}^{2}$ for $\lambda \in P_{+}$.

Proof. Let again $W$ denote the Weyl group of type $B C_{q}$. We introduce the normalized $W$ invariant orbit sums

$$
\widetilde{M}_{\lambda}(x):=\frac{1}{|W \lambda|} \sum_{\mu \in W \cdot \lambda} e^{i\langle\lambda, x\rangle}, \quad \lambda \in P_{+} .
$$

Then the Jacobi polynomials $R_{\lambda}^{p}$ can be written as linear combinations of such orbit sums. It follows from the considerations in [19, Section 11] that for non-negative multiplicity values, the expansion coefficients are all non-negative. That is,

$$
R_{\lambda}^{p}=\sum_{\mu \in P_{+}: \mu \leq \lambda} c_{\lambda \mu} \widetilde{M}_{\mu}
$$


with

$$
c_{\lambda \mu}=c_{\lambda \mu}^{p} \geq 0, \quad \sum_{\mu \leq \lambda} c_{\lambda \mu}=1
$$

Here $\leq$ denotes the dominance order on $P_{+}$given by $\mu \leq \lambda \Longleftrightarrow \sum_{i=1}^{r} \mu_{i} \leq \sum_{i=1}^{r} \lambda_{i}$ for $r=1, \ldots, q$. We have

$$
\partial_{x_{k}} \widetilde{M}_{\lambda}(x)=\frac{1}{|W \lambda|} \sum_{\mu \in W . \lambda} i \mu_{k} e^{i\langle\mu, x\rangle}, \quad \partial_{x_{k} x_{l}} \widetilde{M}_{\lambda}(x)=\frac{-1}{|W \lambda|} \sum_{\mu \in W . \lambda} \mu_{k} \mu_{l} e^{i\langle\mu, x\rangle} .
$$

Notice that $\left|\mu_{k}\right| \leq \lambda_{k} \leq \lambda_{1}$ for each $\mu \in W \lambda$. We thus obtain, independently of $x \in A_{0}$,

$$
\left|\partial_{x_{k}} \widetilde{M}_{\lambda}(x)\right| \leq \lambda_{1}, \quad\left|\partial_{x_{k}} \partial_{x_{l}} \widetilde{M}_{\lambda}(x)\right| \leq \lambda_{1}^{2}
$$

Further, if $\mu \in P_{+}$with $\mu \leq \lambda$, then $\mu_{1} \leq \lambda_{1}$ and therefore

$$
\left|\partial_{x_{k}} R_{\lambda}^{p}(x)\right| \leq \sum_{\mu \leq \lambda} c_{\lambda \mu}\left|\partial_{x_{k}} \widetilde{M}_{\mu}(x)\right| \leq \sum_{\mu \leq \lambda} c_{\lambda \mu} \mu_{1} \leq \lambda_{1}
$$

In the same way,

$$
\left|\partial_{x_{k}} \partial_{x_{l}} R_{\lambda}^{p}(x)\right| \leq \sum_{\mu \leq \lambda} c_{\lambda \mu}\left|\partial_{x_{k}} \partial_{x_{l}} \widetilde{M}_{\mu}(x)\right| \leq \sum_{\mu \leq \lambda} c_{\lambda \mu} \mu_{1}^{2} \leq \lambda_{1}^{2}
$$

We also need some further properties of the moment function $m$. We here have to restrict our attention to the case $p \in\{q, q+1, \ldots, 2 q-1\} \cup] 2 q-1, \infty[$. We shall assume this restriction from now on. We expect that the results below are also valid for all $p \in[q, \infty[$.

\section{Lemma 3.6.}

(1) The matrix $A=\left(a_{r, s}\right)_{r, s=1, \ldots, q} \in M_{q}(\mathbb{R})$ is positive definite.

(2) For all $\lambda \in P_{+} \backslash\{0\}, m(\lambda)>0$.

(3) There exists a constant $C_{1}>0$ such that for all $\lambda \in P_{+}, C_{1} \lambda_{1}^{2} \leq m(\lambda)$.

Proof. For the proof of (1), we first consider the case $p \in] 2 q-1, \infty[$ and conclude from the definition of the $a_{r, s}$ in the proof in Lemma 3.3 that $A$ is symmetric, and that for all $\tau \in \mathbb{R}^{q}$,

$$
\tau^{T} A \tau=\int_{B_{q} \times U(q, \mathbb{F})}\left(\sum_{r=1}^{q} \tau_{r} \operatorname{tr}_{r}\left(\cdot u^{*}\left(w^{*} P_{1}+P_{1} w\right) u\right)\right)^{2} d m_{p}(w) d u,
$$

where the functions

$$
(w, u) \mapsto \operatorname{tr}_{r}\left(\cdot u^{*}\left(w^{*} P_{1}+P_{1} w\right) u\right), \quad B_{q} \times U(q, \mathbb{F}) \rightarrow \mathbb{R},
$$

are linearly independent for $r=1, \ldots q$. This shows that $\tau^{T} A \tau>0$ for all $\tau \in \mathbb{R}^{q}$ with $\tau \neq 0$ as claimed. The case of integers $p \geq q$ can be handled in a similar way by using a modified version of integral representation (3.6) for $a_{r, s}$ which is based on Remark 2.3 instead of Proposition 2.1.

For the proof of part (2) we proceed as in the proof of Lemma 3.5 and write

$$
R_{\lambda}^{p}=\sum_{\mu \in P_{+}: \mu \leq \lambda} c_{\lambda \mu} \widetilde{M}_{\mu} \quad \text { with } \quad c_{\lambda \mu} \geq 0, \quad c_{\lambda \lambda}>0, \quad \sum_{\mu \leq \lambda} c_{\lambda \mu}=1
$$


Thus for $\lambda \in P_{+} \backslash\{0\}$,

$$
m(\lambda)=-\left.\partial_{x_{1}}^{2} R_{\lambda}^{p}(x)\right|_{x=0}=\sum_{\mu \in P_{+}: \mu \leq \lambda} \frac{c_{\lambda \mu}}{|W \mu|} \sum_{\tau \in W \mu} \tau_{1}^{2}>0 .
$$

For the proof of part (3), we use (1) and write $m(\lambda)$ as

$$
m(\lambda)=\lambda^{T} \widetilde{A} \lambda-b^{T} \lambda
$$

with some positive definite matrix $\widetilde{A}$ and some $b \in \mathbb{R}^{q}$. We thus find constants $c, d>0$ such that $m(\lambda)-c \lambda_{1}^{2}>0$ holds for all $\lambda \in P_{+}$with $\lambda_{1} \geq d$. As there are only finitely many $\lambda \in P_{+}$ with $\lambda_{1}<d$, we conclude from part (2) that there exists some $C_{1}>0$ with $m(\lambda)-c \lambda_{1}^{2}>0$ for all $\lambda \in P_{+}$with $\lambda \neq 0$.

Remark 3.7. The nonnegativity of $m(\lambda)$ in Lemma 3.6(2) can be easily established directly. In fact, assume that $m(\lambda)<0$ for some $\lambda \in P_{+}$. Then the Taylor formula

$$
R_{\lambda}(x)=1-\frac{m(\lambda)}{2}\left(x_{1}^{2}+\cdots+x_{q}^{2}\right)+O\left(\|x\|^{3}\right)
$$

implies that $R_{\lambda}(x)>1$ for some $x$ close to 0 , and thus by the Weyl group invariance of $R_{\lambda}$, for some $x \in A_{0}$. But this contradicts the fact that $\left\|R_{\lambda}\right\|_{\infty} \leq 1$ on $A_{0}$, which is a consequence of Proposition 2.1. However, we have no different proof for the strict positivity of $m(\lambda)$ for $\lambda \neq 0$ than the one given in Lemma 3.6(2).

We next use the moment function $m$ in order to define a modified variance of measures $\nu \in M^{1}\left(P_{+}\right)$depending on the underlying convolution $*_{d, p}$. This modified variance will appear in the CLT below.

Definition 3.8. Let $\nu \in M^{1}\left(P_{+}\right)$be a probability measure with finite second moments, meaning that $\sum_{\lambda \in P_{+}} \lambda_{1}^{2} \nu(\{\lambda\})<\infty$. Then the modified second moment $\sigma^{2}:=\sigma^{2}(\nu)$ of $\nu$ is defined as

$$
\sigma^{2}:=\sum_{\lambda \in P_{+}} m(\lambda) \nu(\{\lambda\})
$$

Notice that by Lemmas 3.3 and $3.6, \sigma^{2}$ is finite and non-negative where $\sigma^{2}=0$ holds precisely for $\nu=\delta_{0}$.

Lemma 3.9. Let $\nu \in M^{1}\left(P_{+}\right)$be a probability measure with finite second moments and with the modified variance $\sigma^{2} \geq 0$. Then the spherical Fourier transform

$$
\mathcal{F} \nu: \mathbb{R}^{q} \rightarrow \mathbb{R} \quad \text { with } \quad \mathcal{F} \nu(x):=\sum_{\lambda \in P_{+}} R_{\lambda}^{p}(x) \nu(\{\lambda\})
$$

is twice continuously differentiable on $\mathbb{R}^{q}$ with

$$
\mathcal{F} \nu(0)=1, \quad \nabla \mathcal{F} \nu(0)=0, \quad \text { and Hesse matrix } \quad D^{2}(\mathcal{F} \nu)(0)=-\sigma^{2} I_{q} .
$$

Proof. As $\nu$ has finite second moments, we conclude form Lemma 3.5 that for all $k, l=1, \ldots, q$, the series

$$
\sum_{\lambda \in P_{+}} \nu(\{\lambda\}) \frac{\partial}{\partial x_{k}} R_{\lambda}^{p}(x), \quad \sum_{\lambda \in P_{+}} \nu(\{\lambda\}) \frac{\partial^{2}}{\partial x_{k} \partial x_{l}} R_{\lambda}^{p}(x)
$$

converge uniformly with respect to $x \in \mathbb{R}^{q}$. This implies that $\mathcal{F} \nu(x)$ is twice continuously differentiable on $\mathbb{R}^{q}$, and the partial derivatives commute with the summation. The derivatives at $x=0$ are now obtained by Lemma 3.3. 
We now turn to random walks $\left(S_{n}^{d, p}\right)_{n \geq 0}$ on $\left(P_{+}, *_{d, p}\right)$ associated with some admissible $\nu \in$ $M_{p}^{1}\left(P_{+}\right)$. It is well-known (see, e.g., [3, Section 7.3]) that the additive functional equation for $m$ in Lemma 3.3(3) leads to relations between the modified variance of $\nu$ and random walks associated with $\nu$.

\section{Lemma 3.10.}

(1) For all $\nu_{1}, \nu_{2} \in M_{p}^{1}\left(P_{+}\right)$with finite second moments, the measure $\nu_{1} *_{d, p} \nu_{2} \in M^{1}\left(P_{+}\right)$has also finite second moments, and $\sigma^{2}\left(\nu_{1} *_{d, p} \nu_{2}\right)=\sigma^{2}\left(\nu_{1}\right)+\sigma^{2}\left(\nu_{2}\right)$.

(2) Let $\left(S_{n}^{d, p}\right)_{n \geq 0}$ be a random walk on $\left(P_{+}, *_{d, p}\right)$ associated with the measure $\nu \in M_{p}^{1}\left(P_{+}\right)$with finite second moments. Then, for all integers $n \geq 0$, the expectation of $m\left(S_{n}^{d, p}\right)$ satisfies $E\left(m\left(S_{n}^{d, p}\right)\right)=n \sigma^{2}(\nu)$, and the process $\left(m\left(S_{n}^{d, p}\right)-n \sigma^{2}(\nu)\right)_{n \geq 0}$ is a martingale with respect to the canonical filtration of $\left(S_{n}^{d, p}\right)_{n \geq 0}$.

Proof. Part (1) follows easily from Lemma 3.3(3); c.f. [3, Section 7.3.7]. Moreover, the first assertion of (2) follows from (1) by induction. For the proof of the second statement in (2) we refer to [3, Proposition 7.3.19].

Lemma 3.11. For $a \in] 0, \infty\left[\right.$, define the finite set $K_{a}:=\left\{\lambda \in P_{+}: \lambda_{1} \leq a\right\}$. Let $\left(S_{n}^{d, p}\right)_{n \geq 0}$ be a random walk on $\left(P_{+}, *_{d, p}\right)$ as described above associated with some admissible $\nu \in M_{p}^{1}\left(P_{+}\right)$ with finite second moments. Then, for each $\epsilon>0$ there exists some $a \geq 1$ such that for all $n \in \mathbb{N}, P\left(S_{n}^{d, p} \notin K_{\sqrt{n} a}\right) \leq \epsilon$.

Proof. By Lemma 3.6, we find $c>0$ with $m(\lambda) \geq c \lambda_{1}^{2}$ for $\lambda \in P_{+}$. Therefore, $m(\lambda) \geq c \lambda_{1}^{2} \geq$ $c a^{2} n$ for all $\lambda \in P_{+} \backslash K_{\sqrt{n} a}$ and all $n$. Hence,

$$
P\left(S_{n}^{d, p} \notin K_{\sqrt{n} a}\right) \leq P\left(m\left(S_{n}^{d, p}\right) \geq c a^{2} n\right) \leq \frac{E\left(m\left(S_{n}^{d, p}\right)\right)}{c a^{2} n}=\frac{\sigma^{2} n}{c a^{2} n}=\frac{\sigma^{2}}{c a^{2}}
$$

with the finite modified variance $\sigma^{2}$. This implies the claim.

We are now ready to prove the main result of this section:

Theorem 3.12. Let $p \in\{q, q+1, \ldots, 2 q-1\} \cup] 2 q-1, \infty\left[\right.$. and $\nu \in M_{p}^{1}\left(P_{+}\right)$be an admissible probability measure with $\nu \neq \delta_{0}$ and with finite second moments. Let $\left.\sigma^{2} \in\right] 0, \infty[$ be the modified modified variance of $\nu$, and $\left(S_{n}^{d, p}\right)_{n \geq 0}$ be a random walk on $\left(P_{+}, *_{d, p}\right)$ associated with $\nu$. Then $S_{n}^{d, p} / \sqrt{n \sigma^{2}}$ converges in distribution to the distribution $\rho_{d, p} \in M^{1}(C)$ of a Laguerre ensemble in $C$.

Proof. Fix $x \in C$. Let $n \in \mathbb{N}$ be large enough such that $x / \sqrt{n} \in A_{0}$. By Section $3.2, S_{n}^{d, p}$ has the distribution $\nu^{(n)}$. We thus obtain from the multiplicativity of the spherical Fourier transform of probability measures on $\left(P_{+}, *_{d, p}\right)$, Lemma 3.9 , the qualitative Taylor formula, and from the properties of the moment functions in Lemma 3.3 that for $n \rightarrow \infty$,

$$
\begin{aligned}
E\left(R_{S_{n}^{d, p}}(x / \sqrt{n})\right) & =(\mathcal{F} \nu(x / \sqrt{n}))^{n}=\left(1-\frac{\sigma^{2}}{2 n}\left(x_{1}^{2}+\cdots+x_{q}^{2}\right)+o(1 / n)\right)^{n} \\
& \longrightarrow e^{-\left(x_{1}^{2}+\cdots+x_{q}^{2}\right) \sigma^{2} / 2}
\end{aligned}
$$

Now fix $\epsilon>0$. By Lemma 3.11, there is so $a>0$ such that for all $n \in \mathbb{N}, P\left(S_{n}^{d, p} \notin K_{\sqrt{n} a}\right) \leq \epsilon$. We now conclude from the Mehler-Heine formula (2.4) that for all $\lambda \in K_{\sqrt{n} a}$, that is, $\lambda \in P_{+}$ with $\lambda_{1} \leq a \sqrt{n}$,

$$
\left|R_{\lambda}^{p}(x / \sqrt{n})-\widetilde{\varphi}_{\lambda}^{p}(x / \sqrt{n})\right| \leq C_{1} x_{1}^{2} \lambda_{1} e^{C_{2} x_{1}^{2} \lambda_{1} / \sqrt{n}} \leq \epsilon
$$


whenever $n$ is sufficiently large. As $\left|R_{\lambda}^{p}(x / \sqrt{n})\right| \leq 1$ and $\left|\widetilde{\varphi}_{\lambda}^{p}(x / \sqrt{n})\right| \leq 1$, we thus have

$$
\begin{aligned}
& \left|E\left(R_{S_{n}^{d, p}}(x / \sqrt{n})\right)-E\left(\widetilde{\varphi}_{S_{n}^{d, p}}(x / \sqrt{n})\right)\right| \\
& \quad \leq E\left(\left|R_{S_{n}^{d, p}}(x / \sqrt{n})-\widetilde{\varphi}_{S_{n}^{d, p}}(x / \sqrt{n})\right| \mathbf{1}_{\left\{S_{n}^{d, p} \in K_{\sqrt{n} a}\right\}}\right)+2 P\left(S_{n}^{d, p} \notin K_{\sqrt{n} a}\right) \leq 3 \epsilon
\end{aligned}
$$

for $n$ sufficiently large. Together with (3.7) and the identity $\widetilde{\varphi}_{c y}^{p}(x)=\widetilde{\varphi}_{y}^{p}(c x)$ for $c>0$ and $x, y \in C$, this implies that for all $x \in C$,

$$
\begin{aligned}
\lim _{n \rightarrow \infty} E\left(\widetilde{\varphi}_{S_{n}^{d, p} / \sqrt{\sigma^{2} n}}^{p}(x)\right) & =\lim _{n \rightarrow \infty} E\left(\widetilde{\varphi}_{S_{n}^{d, p}}^{p}\left(x / \sqrt{\sigma^{2} n}\right)\right) \\
& =\lim _{n \rightarrow \infty} E\left(R_{S_{n}^{d, p}}\left(x / \sqrt{\sigma^{2} n}\right)\right)=e^{-\left(x_{1}^{2}+\cdots+x_{q}^{2}\right) / 2}
\end{aligned}
$$

From this limit, equation (3.3) and Levy's continuity theorem for the spherical Fourier transform on the double coset hypergroup $\left(C, \bullet_{d, p}\right)$ (see, e.g., [3, Section 4.2]), we now infer that $S_{n}^{d, p} / \sqrt{\sigma^{2} n}$ converges in distribution to $\rho_{d, p}$ as claimed.

Theorem 1.1 in the introduction is an immediate consequence from Theorem 3.12 and Lemma 3.1.

We also remark that the methods of the preceding proof lead with some additional technical effort to rates of convergence in the CLT; see [11, 29] for the rank one case.

We finish this paper with a strong law of large numbers; it follows easily from the preceding properties of the moment function $m$, in combination with strong laws of large numbers for random walks on commutative hypergroups in [3, Section 7.3] and [32].

Theorem 3.13. Let $\nu \in M_{p}^{1}\left(P_{+}\right)$be admissible with with finite second moments, and let $\left(S_{n}^{d, p}\right)_{n \geq 0}$ be an associated random walk on $\left(P_{+}, *_{d, p}\right)$. Then for all $\epsilon>1 / 2, S_{n} / n^{\epsilon} \rightarrow 0$ almost surely.

Proof. Consider first the hypergroup case with an integer $p \geq q$. By Lemmas 3.3 and 3.6, all conditions of Theorem 7.3.26 in [3] are satisfied for the time-homogeneous random walk $\left(S_{n}^{d, p}\right)_{n \geq 0}$, the sequence $\left(r_{n}:=n^{2 \epsilon}\right)_{n \geq 1}$, and the moment function $m$ instead of $m_{2}$ in [3]. This theorem now yields that $m\left(S_{n}^{d, p}\right) / n^{2 \epsilon}$ tends to 0 almost surely, and Lemma 3.6 proves the claim. An inspection of the details in the proof of Theorem 7.3.26 in [3] shows that this theorem is also available for all $p$ and admissible $\nu \in M_{p}^{1}\left(P_{+}\right)$which proves the theorem in general.

\section{References}

[1] Aslaksen H., Quaternionic determinants, Math. Intelligencer 18 (1996), 57-65.

[2] Ben Saïd S., Ørsted B., Analysis on flat symmetric spaces, J. Math. Pures Appl. 84 (2005), 1393-1426.

[3] Bloom W.R., Heyer H., Harmonic analysis of probability measures on hypergroups, de Gruyter Studies in Mathematics, Vol. 20, Walter de Gruyter \& Co., Berlin, 1995.

[4] Clerc J.L., Une formule asymptotique du type Mehler-Heine pour les zonoles d'un espace riemannien symétrique, Studia Math. 57 (1976), 27-32.

[5] Clerc J.L., Roynette B., Un théorème central-limite, in Analyse harmonique sur les groupes de Lie (Sém., Nancy-Strasbourg, 1976-1978), II, Lecture Notes in Math., Vol. 739, Springer, Berlin, 1979, 122-131.

[6] de Jeu M., Paley-Wiener theorems for the Dunkl transform, Trans. Amer. Math. Soc. 358 (2006), 42254250, math.CA/0404439.

[7] Dunkl C.F., The measure algebra of a locally compact hypergroup, Trans. Amer. Math. Soc. 179 (1973), 331-348.

[8] Eymard P., Roynette B., Marches aléatoires sur le dual de SU(2), in Analyse harmonique sur les groupes de Lie, Lecture Notes in Math., Vol. 497, Editors P. Eymard, J. Faraut, G. Schiffmann, R. Takahashi, Springer-Verlag, Berlin - New York, 1975, 108-152. 
[9] Faraut J., Analyse harmonique sur les paires de Guelfand et les espaces hyperboliques, in Analyse harmonique (Université de Nancy I, 1980), Editors J.L. Clerc, P. Eymard, J. Faraut, M. Raïs, R. Takahasi, Les Cours du C.I.M.P.A., Nice, France, 1983, 315-446.

[10] Faraut J., Korányi A., Analysis on symmetric cones, Oxford Mathematical Monographs, Oxford Science Publications, The Clarendon Press, Oxford University Press, New York, 1994.

[11] Gallardo L., Comportement asymptotique des marches aléatoires associées aux polynômes de Gegenbauer et applications, Adv. in Appl. Probab. 16 (1984), 293-323.

[12] Heckman G., Dunkl operators, Astérisque (1997), exp. No. 828, 223-246.

[13] Heckman G., Schlichtkrull H., Harmonic analysis and special functions on symmetric spaces, Perspectives in Mathematics, Vol. 16, Academic Press, Inc., San Diego, CA, 1994.

[14] Helgason S., Groups and geometric analysis: integral geometry, invariant differential operators, and spherical functions, Mathematical Surveys and Monographs, Vol. 83, Amer. Math. Soc., Providence, RI, 2000.

[15] Jewett R.I., Spaces with an abstract convolution of measures, Adv. Math. 18 (1975), 1-101.

[16] Koornwinder T.H., Two-variable analogues of the classical orthogonal polynomials, in Theory and Application of Special Functions (Proc. Advanced Sem., Math. Res. Center, Univ. Wisconsin, Madison, Wis., 1975), Academic Press, New York, 1975, 435-495.

[17] Koornwinder T.H., Harmonics and spherical functions on Grassmann manifolds of rank two and two-variable analogues of Jacobi polynomials, in Constructive Theory of Functions of Several Variables (Proc. Conf., Math. Res. Inst., Oberwolfach, 1976), Lecture Notes in Math., Vol. 571, Springer, Berlin, 1977, 141-154.

[18] Koornwinder T.H., Positive convolution structures associated with quantum groups, in Probability Measures on Groups, X (Oberwolfach, 1990), Plenum, New York, 1991, 249-268.

[19] Macdonald I.G., Orthogonal polynomials associated with root systems, Sém. Lothar. Combin. 45 (2000), Art. B45a, 40 pages, math.QA/0011046.

[20] Olver F.W.J., Lozier D.W., Boisvert R.F., Clark C.W., NIST handbook of mathematical functions, U.S. Department of Commerce, National Institute of Standards and Technology, Washington, DC; Cambridge University Press, Cambridge, 2010.

[21] Opdam E.M., Dunkl operators, Bessel functions and the discriminant of a finite Coxeter group, Compositio Math. 85 (1993), 333-373.

[22] Opdam E.M., Harmonic analysis for certain representations of graded Hecke algebras, Acta Math. 175 (1995), 75-121.

[23] Remling H., Rösler M., Convolution algebras for Heckman-Opdam polynomials derived from compact Grassmannians, J. Approx. Theory, to appear.

[24] Rösler M., Bessel convolutions on matrix cones, Compos. Math. 143 (2007), 749-779, math.CA/0512474.

[25] Rösler M., Positive convolution structure for a class of Heckman-Opdam hypergeometric functions of type BC, J. Funct. Anal. 258 (2010), 2779-2800, arXiv:0907.2447.

[26] Rösler M., Voit M., Integral representation and uniform limits for some Heckman-Opdam hypergeometric functions of type BC, Trans. Amer. Math. Soc., to appear, arXiv:1402.5793.

[27] Szegö G., Orthogonal polynomials, American Mathematical Society Colloquium Publications, Vol. 23, Amer. Math. Soc., Providence, R.I., 1959.

[28] van Diejen J.F., Properties of some families of hypergeometric orthogonal polynomials in several variables, Trans. Amer. Math. Soc. 351 (1999), 233-270, q-alg/9604004.

[29] Voit M., Central limit theorems for a class of polynomial hypergroups, Adv. in Appl. Probab. 22 (1990), 68-87.

[30] Voit M., Bessel convolutions on matrix cones: algebraic properties and random walks, J. Theoret. Probab. 22 (2009), 741-771, math.CA/0603017.

[31] Voit M., Central limit theorems for hyperbolic spaces and Jacobi processes on [0, $\infty[$, Monatsh. Math. 169 (2013), 441-468, arXiv:1201.3490.

[32] Zeuner H., Moment functions and laws of large numbers on hypergroups, Math. Z. 211 (1992), 369-407. 